\title{
Subjective Well-Being in Centenarians: A Comparison of Japan and the United States
}

\author{
Takeshi Nakagawa, $\mathrm{PhD}^{1,2}$, Jinmyoung Cho, $\mathrm{PhD}^{3,4}$, Yasuyuki Gondo, $\mathrm{PhD}^{5}$, Peter Martin, \\ $\mathbf{P h D}^{6}$, Mary Ann Johnson, $\mathbf{P h D}^{7}$, Leonard W. Poon, PhD $^{8}$, and Nobuyoshi Hirose, PhD $^{9}$ \\ ${ }^{1}$ University Priority Research Program "Dynamics of Healthy Aging”, University of Zurich, Zurich, \\ Switzerland 2JSPS Postdoctoral Fellow for Research Abroad ${ }^{3}$ Center for Applied Health \\ Research, Baylor Scott \& White Health, Temple, Texas ${ }^{4}$ Texas A\&M Health Science Center \\ School of Public Health, College Station, Texas ${ }^{5}$ Graduate School of Human Sciences, Osaka \\ University, Osaka, Japan ${ }^{6}$ Department of Human Development and Family Studies, lowa State \\ University, Ames, lowa ${ }^{7}$ Department of Foods and Nutrition Sciences, University of Georgia, \\ Athens, Georgia ${ }^{8}$ Institute of Gerontology, University of Georgia, Athens, Georgia ${ }^{9}$ Center for \\ Supercentenarian Research, School of Medicine, Keio University, Tokyo, Japan
}

\begin{abstract}
Objective: The present study examined the cultural differences and similarities in the levels and predictors of subjective well-being in Japanese and American centenarians.

Method: We analyzed data on cognitively intact Japanese $(N=59)$ and American $(N=125)$ participants from the Tokyo and Georgia Centenarian Studies, respectively. The Philadelphia Geriatric Center Morale Scale was used to measure subjective well-being, while sociodemographic, social, and health resources were assessed as potential predictors.
\end{abstract}

Results: The American participants reported higher scores on well-being (satisfaction with social relations and psychological comfort). However, cultural differences in levels of well-being disappeared after we controlled for its predictors. The regression models revealed that health resources (cognitive function, hearing problems, and activities of daily living) were strong predictors of well-being in both countries. Social resources (living with others) were strongly associated with one dimension of well-being (attitude toward one's aging) among the Japanese participants.

Discussion: The findings support the existing lifespan and cross-cultural literature, indicating that declines in health impose certain limitations on adaptive capacity in oldest-old age irrespective of cultures, and that social embeddedness is valued in Eastern cultures. The authors speculate that cultural values, i.e., personal autonomy versus relational harmony, play an important role for well-being in oldest-old age.

Address correspondence to Takeshi Nakagawa, University of Zurich, University Priority Research Program "Dynamics of Healthy Aging", Andreasstrasse 15, Box 2, Zurich, 8050, Switzerland. takeshi.nakagawa@ dynage.uzh.ch.

Conflicts Of Interest

No conflicts of interest have been declared by the authors. 


\section{Keywords}

well-being; culture; oldest-old; resources; quality of life

Longevity has been a central theme in research on aging since the 1950s (e.g., Medawar, 1952). In addition, numerous researchers have focused on centenarians for several decades in order to unravel the secrets of exceptional longevity (Andersen et al., 1998; Dello Buono, Urciuoli, \& De Leo, 1998; Evert, Lawler, Bogan, \& Perls, 2003; Wilmoth, Skytthe, Friou, \& Jeune, 1996). Although the majority of previous studies on the oldest-old (i.e., those aged 85 years and over) has accumulated substantial evidence regarding declines in health and functional status, such as cognitive and physical functions (Berlau, Corrada, \& Kawas, 2009; Corrada, Brookmeyer, Paganini-Hill, Berlau, \& Kawas, 2010; Evert et al., 2003; Gondo et al., 2006), few studies have examined how oldest-old individuals feel in daily life, how they adapt to age-related losses, and how they make sense of their own lives. Furthermore, a recent theoretical approach argued that individuals make sense of their own lives through cultural values such as the different emphases on personal autonomy versus relational harmony (Markus \& Kitayama, 1991). However, most of this previous research was conducted in Western cultures, such as North America and Europe, of which a limited number of studies elucidated the cultural differences in aging (Fung, 2013). Therefore, the objective of the present study is two-fold: to investigate what resources impact adaptation in extremely old age; and to further evaluate how cultural contexts shape centenarians' experiences in their everyday lives.

Subjective well-being is defined as individuals' emotional and cognitive evaluations of their lives (Diener, 1984). Researchers have viewed subjective well-being as a general indicator of successful aging that measures the extent to which older people adapt to their lives (Baltes \& Baltes, 1990). Although lifespan theories (Baltes, 1997; Heckhausen \& Schulz, 1995) have proposed that older adults are capable of regulating losses and adapting to surviving into old age, researchers have assumed that age-related losses can severely limit this adaptive capacity, especially in oldest-old age (Baltes \& Smith, 2003; Gerstorf \& Ram, 2009). However, few studies have supported this assumption, indicating that centenarians are less satisfied with their lives than younger people (Dello Buono et al., 1998; Martin, Poon, Kim, \& Johnson, 1996). In contrast, several findings have suggested that subjective well-being remains relatively stable in oldest-old age (Kunzmann, Little, \& Smith, 2000; Martin, Da Rosa, Margrett, Garasky, \& Franke, 2012; Rott \& Jopp, 2006; Wettstein, Schilling, Reidick, $\&$ Wahl, 2015). For instance, longitudinal studies have shown that, as positive affect decreases, negative affect remains stable through oldest-old age (Kunzmann et al., 2000; Martin et al., 2012). Another longitudinal study demonstrated that multiple domains of subjective well-being remained stable or even increased during oldest-old age (Wettstein et al., 2015). These findings challenge the notion that psychological resilience approaches a certain limit and well-being decreases in oldest-old age.

Previous research on the predictors of well-being in old age have suggested that the correlates of well-being can differ across age groups (e.g., Isaacowitz \& Smith, 2003; Jopp, Rott, \& Oswald, 2008; Margrett et al., 2011; Pinquart \& Sörensen, 2000). A significant

Aging Ment Health. Author manuscript; available in PMC 2019 October 01. 
number of studies have addressed the unique characteristics of older individuals through comparisons among young-old, old-old, and oldest-old ages. One meta-analysis suggested that the association between subjective well-being and physical and social resources was stronger in the old-old than in the young-old (Pinquart \& Sörensen, 2000). Researchers acknowledged that evidence on the resources for well-being in oldest-old age was insufficient (Pinquart \& Sörensen, 2000; Smith, Borchelt, \& Maier, 2002), and it is still lacking today.

As an exception, a series of results have been reported from studies of oldest-old adults in Western countries. However, previous findings have been inconsistent. Objective health status had negative influences on well-being through subjective health status among old-old and oldest-old adults (Smith, Fleeson, Geiselmann, Settersten, \& Kunzman, 1999). On the other hand, other studies of the oldest-old reported that physical resources were not associated with well-being (Isaacowitz \& Smith, 2003; Jopp \& Rott, 2006; Margrett et al., 2011). With regard to social resources, one study showed that greater social resources were associated with better scores on multiple well-being measures among centenarians, but with only one well-being measure among octogenarians (Margrett et al., 2011). In contrast, another study found that more social resources were related to better affect in young-old age, but not in oldest-old age (Isaacowitz \& Smith, 2003). Cognitive functions were not a consistent correlate of well-being across or within prior studies (Isaacowitz \& Smith, 2003; Margrett et al., 2011). Cognitive functions may have double-edged effects. In fact, one study of centenarians revealed that, whereas the direct effect of cognition on well-being was negative, cognition had a positive effect through self-efficacy (Jopp \& Rott, 2006).

Given the previous literature, research on well-being and aging has flourished in recent decades, albeit mainly in Western countries. In this regard, since evidence in East Asian countries has remained relatively sparse, the present study aims to fill this gap.

One theory of culture and self (Markus \& Kitayama, 1991) has argued that individuals learn what is important in their lives through cultural contexts, and they internalize cultural values into their personal beliefs. This theory further proposed a conceptual framework for understanding two forms of self-construal: 1) that individuals in Western cultures view themselves as independent and they tend to pursue personal goals; and 2) that individuals in Eastern cultures consider themselves as interdependent and they tend to value relational harmony (Markus \& Kitayama, 1991). Indeed, in line with this theory, cross-cultural studies have indicated that cultural contexts can shape the meanings, levels, and predictors of wellbeing, not only in young adulthood (e.g., Kitayama, Markus, \& Kurokawa, 2000;

Schimmack, Oishi, \& Diener, 2002; Uchida \& Kitayama, 2009), but also in middle and old age (e.g., Karasawa et al., 2011; Kitayama, Karasawa, Curhan, Ryff, \& Markus, 2010; Pethtel \& Chen, 2010).

With respect to the meaning of well-being, a prior study incorporating American and Japanese undergraduate students reported that positive emotions were associated with personal achievement in the United States and social harmony in Japan (Uchida \& Kitayama, 2009), which indicates that internal aspects of well-being are valued in Western cultures, whereas external aspects of well-being are emphasized in Eastern cultures. In 
addition, Schimmack, Oishi, and Diener (2002) suggested that, whereas people in Western cultures perceive emotions as opposite-valence ones (i.e., positive versus negative) and they are motivated to maximize their positive emotions, those in Eastern cultures have dialectic beliefs on emotions and they are predisposed to experience a balance between positive and negative emotions in order to adjust to their respective environments.

Regarding the cultural differences in the mean levels and predictors of well-being, Karasawa et al. (2011) demonstrated that mean levels of overall well-being were higher among middleaged Americans than their Japanese counterparts, but the differences were less evident in old age. Furthermore, Kitayama et al. (2010) reported that the strongest predictor of well-being was personal control in the United States, while such a predictor was the absence of relational strain in Japan. Although researchers have begun to focus on cultural differences in aging (Fung, 2013), little is known about how cultural contexts shape the daily well-being of individuals and moderate the links between well-being and resources in oldest-old age.

As stated earlier, the present study investigates the cross-cultural differences and similarities in the mean levels and predictors of subjective well-being among Japanese and American centenarians. For this purpose, we adapt the lifespan and cross-cultural perspectives, and extend previous empirical research (e.g., Isaacowitz \& Smith, 2003; Karasawa et al., 2011; Kitayama et al., 2010; Margrett et al., 2011) to oldest-old age. Overall, two research questions are examined in the present study. First, we test the mean-level differences in subjective well-being between Japan and the United States. We hypothesize that American centenarians would exhibit higher levels of subjective well-being than their Japanese counterparts because, whereas individuals in Western cultures are likely to enhance their positive emotions, those in Eastern cultures are motivated to suppress personal emotions so as not to disrupt social harmony (Markus \& Kitayama, 1991). Previous studies have indicated that the mean levels of cognitive and affective components of subjective well-being can differ across cultures (Karasawa et al., 2011; Pethtel \& Chen, 2010). Thus, multidimensional well-being is assessed in the current study. Second, this study investigates the cultural differences in the predictors (i.e., social and health resources) of well-being between the two aforementioned countries. Since social harmony and embeddedness are highly valued in Eastern cultures (Markus \& Kitayama, 1991), we also predict that social resources are more related to well-being among the Japanese centenarians, compared to their American counterparts. Our study used coresidence with others and living at a familiar place (i.e., one's own house) as social variables, given the broader conceptual framework encompassing the social and physical environment (e.g., social networks and housing) (Wahl \& Lang, 2004). Regarding health resources, although lifespan theories have proposed that older adults have the capacity to adapt to aging (Baltes, 1997; Heckhausen \& Schulz, 1995), age-related limitations in health can impose self-regulatory capacity for managing losses, particularly in oldest-old age (Baltes \& Smith, 2003; Gerstorf \& Ram, 2009). Based on the lifespan perspective, we predicted that general declines in health would be associated with lower levels of well-being in both Japanese and American centenarians. Hence, the present study facilitates the understanding of cultural variation among extremely long-lived individuals' experiences in daily life and highlights the important resources for adaptation. 


\section{Method}

\section{Participants}

This study used data from two independent studies in Japan and the United States (Gondo et al., 2006; Poon et al., 1992). First, the Japanese sample was derived from the Tokyo Centenarian Study (Gondo et al., 2006). A total of 1,194 centenarians living in the 23 wards of metropolitan Tokyo were selected via the residential list. This population-based study sample included 304 centenarians who participated in a mail-based survey and a subsequent visit survey. Only the cognitively intact participants were asked to answer the survey questions. Second, the American sample was from Phase I of the Georgia Centenarian Study (Poon et al., 1992). A sample of 137 centenarians and near-centenarians (99 years and older) living in the state of Georgia were recruited. The inclusion criteria were that all of the participants were required to be cognitively intact. The unique feature of the two comparison samples was that all of the participants were cognitively intact, which enabled them to respond to the survey questions on health and well-being.

\section{Procedure}

The Global Deterioration Scale (GDS) (Reisberg, Ferris, De Leon, \& Crook, 1982) was applied to both studies in order to exclude any participants with cognitive impairments. The GDS was developed to assess the severity of dementia, based on behavioral observations by researchers and practitioners in which a higher score indicates more severe cognitive impairments. In addition, the proposed cutoff score for dementia was four (American Psychiatric Association, 2007). The eligible number of samples totaled 92 in Japan and 135 in the United States. After excluding the participants with missing data for self-report measures, the final sample in the present study consisted of 59 Japanese and 125 American individuals who completed the cognitively demanding assessments. Eight (6.4\%) Americans were 99 years old. The Japanese and American studies were approved by the ethical committees of the Keio University School of Medicine and the University of Georgia Institutional Review Board on Human Subjects, respectively.

\section{Measures}

Well-Being-Subjective well-being was assessed with the 17-item Philadelphia Geriatric Center Morale Scale (PGCMS; Lawton, 1975). The participants were asked to make a choice between two responses such as yes or no, better or worse, satisfied or not, and not much or a lot. The PGCMS consists of three factors: attitude toward one's aging, satisfaction with social relations, and psychological comfort. The five items on the attitude toward one's aging (e.g., "Things keep getting worse as I get older") capture the individuals' perception of changes in their lives. The six items on satisfaction with social relations (e.g., "How much do you feel lonely?") encompass satisfaction with social interactions in everyday life. The six items on psychological comfort (e.g., "Are you afraid of a lot of things?") represent general anxiety. Overall, the factor structure was consistent and comparable between Japan and the United States (Liang, Asano, Bollen, Kahana, \& Maeda, 1987). Initially, attitude toward one's aging was interpreted as cognitive well-being, and satisfaction with social relations and psychological comfort were regarded as affective well-being (Lawton, 1975).

Aging Ment Health. Author manuscript; available in PMC 2019 October 01. 
Moreover, the negative items were reverse-coded and the higher scores indicated better subjective well-being.

One item with a low Cronbach's alpha coefficient was excluded (i.e., the psychological comfort item "Do little things bother you more this year?"). Then, 16 items of the PGCMS were used in the subsequent analyses. The revised total PGCMS score showed a high Cronbach's alpha coefficient. The internal consistency slightly decreased from $a=.73$ to $a$ $=.72$ for Japan, whereas it improved from $a=.69$ to $a=.77$ for the United States.

Predictors-Sociodemographic, social, and health variables were measured as potential predictors of subjective well-being. Age (years), gender ( $O=$ male, $1=$ female), and education (years of schooling were converted into $1=0-6$ years, $2=7-9$ years, $3=10-12$ years, $4=13-15$ years, $5=16$ years and over) were included as sociodemographic predictors, while two variables were assessed as social predictors. In regard to the latter two variables, the participants were asked whether they lived in their own private homes or if they lived alone ( $O=n o, 1=$ yes for both variables). If the participants answered "no" to the former question, then they were considered to be living in an unfamiliar place such as a hospital or a care facility. In addition, five indicators were measured as health predictors: cognitive function, vision problem, hearing problem, number of diseases, and activities of daily living (ADL).

The GDS was also administered to evaluate cognitive function. Vision and hearing problems were reported ( $O=$ absent, $1=$ present for both variables), and the participants were asked whether they had the following nine diseases $(O=n o, 1=y e s$ for each disease): stroke, dysfunction, myocardial infarction, Parkinson's disease, high blood pressure, diabetes mellitus, cancer, cataract, and fractures. Then, the number of diseases was calculated. Finally, ADL was measured as the total number of the six items that the participants were able to perform on their own (Fillenbaum, 1985): eating, dressing, walking, getting in and out of bed, taking a bath, and going to the bathroom $(O=$ completely unable, $1=$ with some help, $2=$ no problem for each item). Cronbach's alpha coefficients regarding ADL were .71 for the Japanese participants and .64 for the American participants.

\section{Statistical Analysis}

This statistical analysis proceeded in three steps. First, we compared the key variables between the two countries using $t$-tests and chi-square tests to examine the differences in subjective well-being and its potential predictors. Given the high proportion of dropouts in the Japanese sample (35.9\%; $7.4 \%$ in the American sample), follow-up analyses were performed to examine the patterns of missing data for self-reports. Second, we computed zero-order correlations between subjective well-being and its predictors for each country. Third, multiple hierarchical regression analyses were performed on the total PGCMS score and each of the three factors. Because of the small sample size, we included variables exceeding the minimum correlation coefficients $(r= \pm .05)$ in either of the two countries as predictors in the regression models. Using the combined Japan and the United States sample, sociodemographic variables, including culture ( $O=$ the United States, $1=$ Japan), were entered first, followed by social and health variables (continuous variables were mean- 
centered for further analyses). The interactions between culture and predictors were entered in the final step to examine the differences in the predictive patterns of subjective wellbeing. The analyses were conducted via IBM SPSS Statistics for Windows Version 23, and the significance level was adopted at .05 .

\section{Results}

\section{Mean Sample Differences}

Significant differences in subjective well-being and the potential predictors were found between the Japanese and American centenarians (see Table 1). With respect to subjective well-being, the total PGCMS score did not differ between Japan and the United States. However, country differences were found in two of the three factors on the PGCMS. Although there was no significant difference in the attitude toward one's aging between the two countries, the satisfaction with social relations and psychological comfort were higher among the Americans compared to the Japanese.

Regarding predictors of well-being, we found significant country differences in age and on all social and health variables. The Japanese were a half-year older than the Americans. With respect to the social variables, more Japanese participants lived in their own homes or with others, compared to the American participants. Regarding the health variables, differential patterns were observed. Sensory functions and diseases were better among the Americans than the Japanese. The American centenarians also reported vision and hearing problems less frequently than their Japanese counterparts. Meanwhile, the number of diseases was lower among the American participants, compared to the Japanese participants. Conversely, cognitive and physical functions (assessed by GDS and ADL, respectively) were better among the Japanese, compared to the Americans.

To examine the patterns of missing data on self-reports, we performed chi-square tests separately for each country to compare distributions of the categorical variables between individuals with and without missing data, and 2 (country) $\times 2$ (missing data) ANOVAs were conducted to compare mean values for the continuous variables. The two-way interactions were significant for age and ADL: $F(1,223)=9.22, p<.01$, and $F(1,220)=6.12, p=.01$, respectively. American, but not Japanese, participants with missing data were older than those without missing data $(M=102.50, S D=2.22$ for the Americans with missing data). Japanese, but not American, participants with missing data reported a lower level of ADL than those without missing data $(M=7.97, S D=3.56$ for the Japanese with missing data).

\section{Intercorrelations Between the Variables}

The intercorrelations between the subjective well-being indicators and the potential predictors were separately calculated for Japan and the United States (see Table 2). Whereas none of the sociodemographic variables were significantly correlated in either country, most of the health variables, except for vision problems and the number of diseases, were significantly associated with subjective well-being in the United States. Only hearing problems were significantly correlated with subjective well-being in both countries. One social predictor was associated with subjective well-being among the Japanese participants, 
but not among the American participants: living alone was negatively related with the attitude toward one's aging in Japan.

Because the sizes of the correlations obtained above were greater than \pm .05 , we included all the variables in the subsequent regression analyses. To test whether the social and health predictors of well-being differed between Japan and the United States, we used the sociodemographic predictors as the control variables and the social and health predictors as the independent variables, and then we entered interaction terms between the independent variables and culture.

\section{Regression Predicting Subjective Well-Being}

Multiple hierarchical regression analyses revealed cross-country differences as well as similarities in the predictive patterns of subjective well-being. The results from the regression analyses are summarized in Table 3 . The unique variance explained by the sociodemographic variables was significant only with regard to satisfaction with social relations. Moreover, culture was not significantly associated with either satisfaction with social relations or psychological comfort, after controlling for the potential predictors. These results indicate that country differences in the levels of the two subjective well-being indicators (shown in Table 1) can be explained by the predictors.

The social variables, when entered as a set of predictors, did not explain the variances in the regression models, and none of the regression coefficients were significant. In contrast, the health variables, when entered into the regression models, significantly explained the variances of the subjective well-being indicators, except for attitude toward one's aging. Overall, better health variables predicted higher levels of well-being. Lower cognitive function was associated with a lower level of psychological comfort. Hearing problems had a negative effect on the subjective well-being indicators. Furthermore, ADL was found to be a significant predictor of the total PGCMS score and the satisfaction with social relations.

Regarding the moderation effect of culture on the relationship between subjective well-being and its potential predictors, the interaction terms failed to improve model fits with respect to any of the well-being indicators. However, a significant interaction was found between culture and living alone in predicting the attitude toward one's aging. According to Figure 1, which illustrates the effect of social resources on subjective well-being in Japan and the United States, Japanese centenarians living alone reported a lower score in the attitude toward one's aging than their counterparts living with others. In contrast, there was no difference in the subjective well-being indicator for American centenarians, irrespective of whether they lived alone.

\section{Discussion}

In order to enhance the understanding regarding the well-being of oldest-old individuals in different cultural contexts, the present study examined whether the mean levels and the predictors of subjective well-being differ between Japanese and American centenarians. Based on the lifespan and cross-cultural perspectives, it was anticipated that cultural differences and similarities would be observed. 
This study tested the first hypothesis that older American adults would exhibit higher levels of subjective well-being than their Japanese counterparts. As predicted, the satisfaction with social relations and psychological comfort were higher among the Americans, compared to the Japanese participants. In contrast, there was no difference in the attitude toward one's aging between the two countries. These results partially support the cross-cultural theory, which assumes that, while individuals in Western cultures (i.e., the United States) view themselves as independent, able to pursue personal goals, and are likely to maximize their positive emotions, individuals in Eastern cultures (i.e., Japan) perceive themselves as interdependent, able to secure social harmony, and are likely to suppress their emotions so as not to threaten social relationships (Markus \& Kitayama, 1991). As for the same degree of the attitude toward one's aging, Japanese centenarians might internalize traditional cultural values regulating interpersonal relations between older and younger people (i.e., filial piety) (Takagi \& Silverstein, 2006) into their personal beliefs and exhibit positive attitudes toward aging. Indeed, if older Japanese adults conformed to the traditional cultural norms, a negative influence of support from a child on their subjective well-being was buffered (Takagi \& Saito, 2013). However, the recent increase in the aging population of Eastern cultures has threatened traditional cultural values, and older adults' attitudes toward aging were more negative in the East than in the West (North \& Fiske, 2015). In future, older adults in Japan may hold increasingly negative attitudes toward aging.

The regression analyses further revealed the cultural differences as well as the similarities in the predictive resources for subjective well-being in oldest-old age (see Table 3). The findings support the second hypothesis. As expected, the health predictors largely explained the subjective well-being indicators in both countries, except for attitude toward one's aging. These results are congruent with prior findings regarding the positive association between well-being and health resources in young-old, old-old, and oldest-old people (Pinquart \& Sörensen, 2000; Smith et al., 1999). Health was a universal predictor of well-being in old age, and age-related declines in health can impose certain limitations on adaptive capacity in oldest-old age, regardless of the cultural contexts.

The present study also found different predictive effects of health resources on each component of well-being. More specifically, while physical impairment (i.e., ADL) predicted lower degrees of satisfaction with social relations, cognitive and hearing impairments were negatively associated with psychological comfort. Contrary to previous studies reporting neutral effects of physical functions and mixed influences of cognitive functions on well-being (Isaacowitz \& Smith, 2003; Jopp \& Rott, 2006; Margrett et al., 2011), the present study found significant effects of physical functions and a beneficial influence of cognitive functions.

These inconsistent results may be due to potential psychological resources. Although previous studies (Isaacowitz \& Smith, 2003; Jopp \& Rott, 2006; Margrett et al., 2011) measured personality traits (i.e., neuroticism and extraversion), the present study did not assess these traits. In this regard, since psychological resources can have a strong impact on affect, it might have diminished the effects of health resources in prior studies. Regarding the negative effect of hearing impairment, the results of the present study were inconsistent with previous research. More specifically, whereas some previous studies suggested that 
hearing impairment can cause negative outcomes, such as reduced physical and social functions (Dalton et al., 2003; Strawbridge, Wallhagen, Shema, \& Kaplan, 2000), a recent study reported that oldest-old individuals with vision impairment displayed worse mental health than their counterparts with hearing impairment (Cimarolli \& Jopp, 2014). Given the present and previous findings, further evidence should be sought to address these inconsistent results as well as the underlying pathways of various aspects of health (e.g., physical, cognitive, and sensory impairments) on well-being in oldest-old age.

Next, social resources were more strongly related to at least one dimension of well-being in Japan than in the United States: the Japanese participants living with others reported a more favorable attitude toward one's aging (see Figure 1). A previous cross-cultural study indicated that social factors would be more salient predictors of well-being among the Japanese than the American participants (Kitayama et al., 2010). Moreover, coresidence with others in old age has been shown to be a desirable situation that also accommodates personal values in Eastern cultures (Takagi \& Silverstein, 2006). In this regard, the Japanese centenarians living with others (mostly with their children) reported a higher level of wellbeing than their counterparts living alone.

Finally, it is important to point out that health resources had strong effects on well-being and they explained the cultural differences related to well-being. Specifically, the regression analyses demonstrated that culture was not significantly related to satisfaction with social relations and psychological comfort, after controlling for the health predictors. Further, cultural differences in health might be partially explained by changes in social policies. Health expenditures have continued to increase since the 1960s in the United States, but leveled off in Japan in the 1980s (Ikegami \& Campbell, 1995), which could cause a longterm and delayed effect on population health. Whereas the proportion of oldest-old Americans without disability has continued to decrease since the 1980s (Manton, Gu, \& Lamb, 2006), the ratio of their Japanese counterparts in poor health decreased during the 1980s, except among women, but has increased since the 1990s (Yong \& Saito, 2009).

Several limitations should be considered in interpreting the findings. First, any generalization of the results should be interpreted with caution. Since this study selected cognitively intact older adults and deleted a high proportion of Japanese cases with missing values due to physical impairments, the findings can only be generalized to healthy oldestold individuals. In addition, although the present study focused on the cultural differences between Japan (i.e., the East) and the United States (i.e., the West), it might not be strictly representative of each culture. Second, psychological variables must be reconsidered. The three factors of the PGCMS empirically overlap (Okun \& Stock, 1987). For example, satisfaction with social relations includes two items on social relations and four items on life satisfaction (Lawton, 1975). Distinct components of well-being should be measured. Further, the robustness of health predictors remains unclear after controlling for personality traits. Third, this study might assess a limited scope of social resources. In our results, the social variables did not significantly explain variances of the regression models, and living at home did not predict well-being in either country. These variables focused on objective status, but they did not assess subjective meanings such as perceived support and attachment to place. In addition, macro-level social resources, such as social policies, should be taken 
into account. The Japanese government implemented its long-term care system in 2000. Thus, it is possible that the provision of long-term care buffers the negative impact of poor health in Japanese centenarians.

In conclusion, the present study highlighted the role of age and culture on well-being in oldest-old age. As for the cultural differences in the mean level of well-being, this study suggests that cultural contexts can shape how older people view aging and how they feel in their everyday lives. With regard to cultural variation in the predictors of well-being, the findings indicate that health resources are critical to the well-being of oldest-old adults, irrespective of cultural contexts. In addition, physical, cognitive, and sensory (i.e., hearing) functions enabled the centenarians in this study to adapt to their lives. Therefore, the results support the lifespan perspective by proposing that age-related losses impose certain limitations on adaptive capacity in oldest-old age. Moreover, the findings suggest that social resources are important in Eastern cultures, and that cultural values play an important role in maintaining one dimension of well-being in oldest-old age. However, researchers need to focus further attention on how oldest-old individuals adapt to losses with advancing age across various cultural contexts.

\section{Acknowledgments}

We would like to thank Dr. Hiroki Inagaki for creating the codebook for the Tokyo Centenarian Study.

Funding

This research was funded by the program for Promoting the Enhancement of Research Universities from Osaka University, Osaka, Japan, and by a grant from the Japan Foundation for Aging and Health.

The Georgia Centenarian Study (Phase I) was supported by R01-MH43435 (1988-92) from the National Institute of Mental Health (L.W. Poon, PI).

The Tokyo Centenarian Study was supported in part by a grant from the Japanese Ministry of Health and Welfare for the Scientific Research Project on Longevity, which focuses on the multidisciplinary approach to centenarians and its international comparison (N. Hirose, PI); a grant from the Japanese Ministry of Education, Science and Culture (No.15730346); and financial aid for research from the Keio Health Consulting Center.

\section{References}

American Psychiatric Association. (2007). Practice guideline for the treatment of patients with Alzheimer's disease and other dementias. Arlington: American Psychiatric Association.

Andersen HR, Jeune B, Nybo H, Nielsen JB, Andersen-Ranberg K, \& Grandjean P (1998). Low activity of superoxide dismutase and high activity of glutathione reductase in erythrocytes from centenarians. Age and Ageing, 27, 643-648. doi:10.1093/ageing/27.5.643. [PubMed: 12675104]

Baltes PB (1997). On the incomplete architecture of human ontogeny: Selection, optimization, and compensation as foundation of developmental theory. American Psychologist, 52(4), 366-380. doi: 10.1037/0003-066X.52.4.366. [PubMed: 9109347]

Baltes PB, \& Baltes MM (1990). Psychological perspectives on successful aging: The model of selective optimization with compensation In Baltes PB \& Baltes MM (Eds.), Successful aging: Perspectives from behavioral sciences (pp. 1-34). New York: Cambridge University Press.

Baltes PB, \& Smith J (2003). New frontiers in the future of aging: From successful aging of the young old to the dilemmas of the fourth age. Gerontology, 49, 123-135. doi:10.1159/000067946 [PubMed: 12574672] 
Berlau DJ, Corrada MM, \& Kawas C (2009). The prevalence of disability in the oldest-old is high and continues to increase with age: Findings from The 90+ Study. International Journal of Geriatric Psychiatry, 24, 1217-1225. doi:10.1002/gps.2248. [PubMed: 19259982]

Cimarolli VR, \& Jopp DS (2014). Sensory impairments and their associations with functional disability in a sample of the oldest-old. Quality of Life Research, 23, 1977-1984. doi:10.1007/ s11136-014-0657-0. [PubMed: 24682668]

Corrada MM, Brookmeyer R, Paganini-Hill A, Berlau D, \& Kawas CH (2010). Dementia incidence continues to increase with age in the oldest old: The 90+ study. Annals of Neurology, 67, 114-121. doi:10.1002/ana.21915. [PubMed: 20186856]

Dalton DS, Cruickshanks KJ, Klein BEK, Klein R, Wiley TL, \& Nondahl DM (2003). The impact of hearing loss on quality of life in older adults. The Gerontologist, 43, 661-668. doi:10.1093/geront/ 43.5.661. [PubMed: 14570962]

Dello Buono M, Urciuoli O, \& De Leo D (1998). Quality of life and longevity: A study of centenarians. Age and Ageing, 27, 207-216. doi:10.1093/ageing/27.2.207. [PubMed: 16296681]

Diener E (1984). Subjective well-being. Psychological Bulletin, 95(3), 542-575. doi: 10.1037/0033-2909.95.3.542. [PubMed: 6399758]

Evert J, Lawler E, Bogan H, \& Perls T (2003). Morbidity profiles of centenarians: Survivors, delayers, and escapers. The Journals of Gerontology Series A: Biological Sciences and Medical Sciences, 58, M232-M237. doi:10.1093/gerona/58.3.M232.

Faul F, Erdfelder E, Buchner A, \& Lang A-G (2009). Statistical power analyses using G*Power 3.1: Tests for correlation and regression analyses. Behavior Research Methods, 41, 1149-1160. doi: 10.3758/BRM.41.4.1149. [PubMed: 19897823]

Fillenbaum GG (1985). Screening the elderly. A brief instrumental activities of daily living measure. Journal of the American Geriatrics Society, 33, 698-706. doi:10.1111/j. 1532-5415.1985.tb01779.x. [PubMed: 4045087]

Fung HH (2013). Aging in culture. The Gerontologist, 53, 369-377. doi:10.1093/geront/gnt024. [PubMed: 23585454]

Gerstorf D, \& Ram N (2009). Limitations on the importance of self-regulation in old age. Human Development, 52, 38-43. doi:10.1159/000189214. [PubMed: 23729835]

Gondo Y, Hirose N, Arai Y, Inagaki H, Masui Y, Yamamura K, ... Kitagawa K (2006). Functional status of centenarians in Tokyo, Japan: Developing better phenotypes of exceptional longevity. The Journals of Gerontology Series A: Biological Sciences and Medical Sciences, 61, 305-310. doi: 10.1093/gerona/61.3.305.

Heckhausen J, \& Schulz R (1995). A life-span theory of control. Psychological Review, 102(2), 284 304. doi:10.1037/0033-295X.102.2.284. [PubMed: 7740091]

Ikegami N, \& Campbell JC (1995). Medical care in Japan. The New England Journal of Medicine, 333, 1295-1299. doi:10.1056/NEJM199511093331922. [PubMed: 7566019]

Isaacowitz DM, \& Smith J (2003). Positive and negative affect in very old age. The Journals of Gerontology Series B: Psychological Sciences and Social Sciences, 58, P143-P152. doi:10.1093/ geronb/58.3.P143.

Jopp D, \& Rott C (2006). Adaptation in very old age: Exploring the role of resources, beliefs, and attitudes for centenarians' happiness. Psychology and Aging, 21(2), 266-280. doi: 10.1037/0882-7974.21.2.266. [PubMed: 16768574]

Jopp D, Rott C, \& Oswald F (2008). Valuation of life in old and very old age: The role of sociodemographic, social, and health resources for positive adaptation. The Gerontologist, 48, 646-658. doi:10.1093/geront/48.5.646. [PubMed: 18981281]

Karasawa M, Curhan KB, Markus HR, Kitayama S, Love GD, Radler BT, \& Ryff CD (2011). Cultural perspectives on aging and well-being: A comparison of Japan and the United States. The International Journal of Aging and Human Development, 73, 73-98. doi:10.2190/AG.73.1.d. [PubMed: 21922800]

Kitayama S, Karasawa M, Curhan KB, Ryff CD, \& Markus HR (2010). Independence and interdependence predict health and wellbeing: Divergent patterns in the United States and Japan. Frontiers in Psychology, 1, 1-10. doi:10.3389/fpsyg.2010.00163. [PubMed: 21833184] 
Kitayama S, Markus HR, \& Kurokawa M (2000). Culture, emotion, and well-being: Good feelings in Japan and the United States. Cognition and Emotion, 14, 93-124. doi:10.1080/026999300379003.

Kunzmann U, Little TD, \& Smith J (2000). Is age-related stability of subjective well-being a paradox? Cross-sectional and longitudinal evidence from the Berlin Aging Study. Psychology and Aging, 15, 511-526. doi:10.1037/0882-7974.15.3.511. [PubMed: 11014714]

Lawton MP (1975). The Philadelphia Geriatric Center Morale Scale: A revision. Journal of Gerontology, 30, 85-89. doi:10.1093/geronj/30.1.85. [PubMed: 1109399]

Liang J, Asano H, Bollen KA, Kahana EF, \& Maeda D (1987). Cross-cultural comparability of the Philadelphia Geriatric Center Morale Scale: An American-Japanese comparison. Journal of Gerontology, 42, 37-43. doi:10.1093/geronj/42.1.37. [PubMed: 3794194]

Manton KG, Gu X, \& Lamb VL (2006). Change in chronic disability from 1982 to 2004/2005 as measured by long-term changes in function and health in the U.S. elderly population. Proceedings of the National Academy of Sciences, 103(48), 18374-18379. doi:10.1073/pnas.0608483103.

Margrett JA, Daugherty K, Martin P, MacDonald M, Davey A, Woodard JL, ... Poon LW (2011). Affect and loneliness among centenarians and the oldest old: The role of individual and social resources. Aging and Mental Health, 15, 385-396. doi:10.1080/13607863.2010.519327. [PubMed: 21491224]

Markus HR, \& Kitayama S (1991). Culture and the self: Implications for cognition, emotion, and motivation. Psychological Review, 98, 224-253. doi:10.1037/0033-295X.98.2.224.

Martin P, Da Rosa G, Margrett JA, Garasky S, \& Franke W (2012). Stability and change in affect among centenarians. The International Journal of Aging and Human Development, 75, 337-349. article. doi:10.2190/AG.75.4.c. [PubMed: 23420992]

Martin P, Poon LW, Kim E, \& Johnson MA (1996). Social and psychological resources in the oldest old. Experimental Aging Research, 22, 121-139. doi:10.1080/03610739608254002. [PubMed: 8735148]

Medawar PB (1952). An unsolved problem of biology. London, UK: HK Lewis.

North MS, \& Fiske ST (2015). Modern attitudes toward older adults in the aging world: A crosscultural meta-analysis. Psychological Bulletin, 141, 993-1021. doi:10.1037/a0039469. [PubMed: 26191955]

Okun MA, \& Stock WA (1987). Correlates and components of subjective well-being among the elderly. Journal of Applied Gerontology, 6(1), 95-112. doi:10.1177/073346488700600108.

Pethtel O, \& Chen Y (2010). Cross-cultural aging in cognitive and affective components of subjective well-being. Psychology and Aging, 25, 725-729. doi:10.1037/a0018511. [PubMed: 20853976]

Pinquart M, \& Sörensen S (2000). Influences of socioeconomic status, social network, and competence on subjective well-being in later life: A meta-analysis. Psychology and Aging, 15, 187-224. doi: 10.1037/0882-7974.15.2.187. [PubMed: 10879576]

Poon LW, Clayton GM, Martin P, Johnson MA, Courtenay BC, Sweaney AL, ... Thielman SB (1992). The Georgia Centenarian Study. The International Journal of Aging and Human Development, 34, 1-17. doi:10.2190/8M7H-CJL7-6K5T-UMFV [PubMed: 1737657]

Reisberg B, Ferris SH, De Leon MJ, \& Crook T (1982). The Global Deterioration Scale for assessment of primary degenerative dementia. American Journal of Psychiatry, 139, 1136-1139. doi:10.1176/ ajp.139.9.1136. [PubMed: 7114305]

Rott C, \& Jopp D (2006). Subjective well-being in centenarians. Global Ageing, 4, 52-62.

Schimmack U, Oishi S, \& Diener E (2002). Cultural influences on the relation between pleasant emotions and unpleasant emotions: Asian dialectic philosophies or individualism-collectivism? Cognition and Emotion, 16, 705-719. doi:10.1080/02699930143000590.

Smith J, Borchelt M, \& Maier H (2002). Health and well-being in the young old and oldest old. Journal of Social Issues, 58(4), 715-732. doi:10.1111/1540-4560.00286.

Smith J, Fleeson W, Geiselmann B, Settersten RAR, \& Kunzman U (1999). Sources of well-being in very old age In Baltes P \& Mayer K (Eds.), The Berlin Aging Study: Aging from 70 to 100 (pp. 450-471). Cambridge, England: Cambridge University Press.

Strawbridge WJ, Wallhagen MI, Shema SJ, \& Kaplan GA (2000). Negative consequences of hearing impairment in old age: A longitudinal analysis. The Gerontologist, 40, 320-326. doi:10.1093/ geront/40.3.320. [PubMed: 10853526] 
Takagi E, \& Saito Y (2013). A longitudinal analysis of the impact of family support on the morale of older parents in Japan: does the parent's normative belief in filial responsibilities make a difference? Ageing and Society, 33(6), 1053-1076. doi:10.1017/S0144686X1200044X. [PubMed: 23913993]

Takagi E, \& Silverstein M (2006). Intergenerational coresidence of the Japanese elderly: Are cultural norms proactive or reactive? Research on Aging, 28(4), 473-492. doi: $10.1177 / 0164027506287788$.

Uchida Y, \& Kitayama S (2009). Happiness and unhappiness in east and west: Themes and variations. Emotion, 9, 441-456. doi:10.1037/a0015634. [PubMed: 19653765]

Wahl HW, \& Lang FR (2004). Aging in context across the adult life course: Integrating physical and social environmental research perspectives In Wahl HW, Scheidt R, \& Windley PG (Eds.), Aging in context: Socio-physical environments. Annual Review of Gerontology and Geriatrics, Vol. 23 (pp. 1-33). New York: Springer.

Wettstein M, Schilling OK, Reidick O, \& Wahl HW (2015). Four-year stability, change, and multidirectionality of well-being in very-old age. Psychology and Aging, 30, 500-516. doi: 10.1037/pag0000037. [PubMed: 26098169]

Wilmoth J, Skytthe A, Friou D, \& Jeune B (1996). The oldest man ever? A case study of exceptional longevity. The Gerontologist, 36, 783-788. doi:10.1093/geront/36.6.783. [PubMed: 8990590]

Yong V, \& Saito Y (2009). Trends in healthy life expectancy in Japan: 1986-2004. Demographic Research, 20, 467-494. doi:10.4054/DemRes.2009.20.19. 


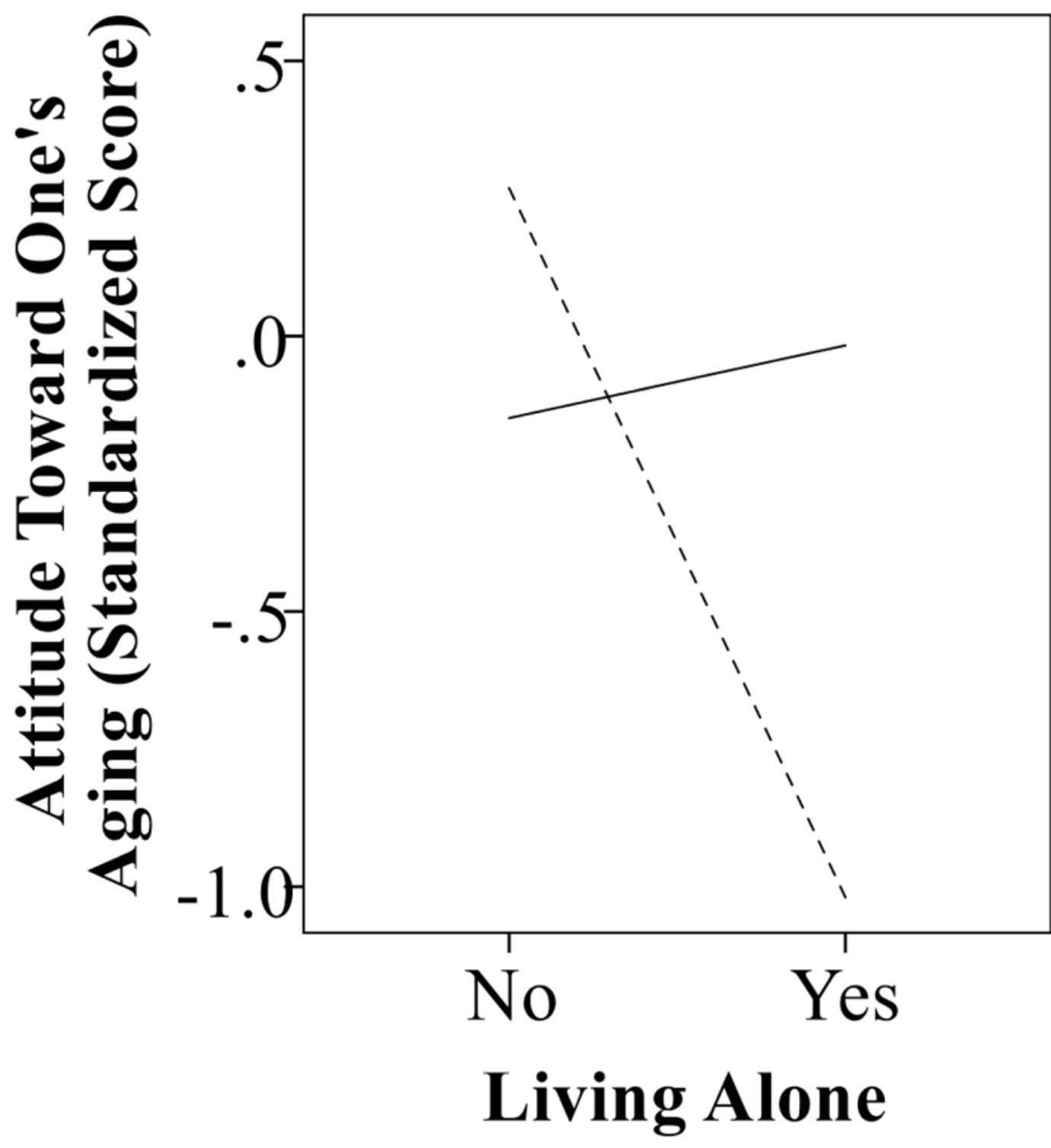

-US
---Japan

Figure 1.

The effect of living status on subjective well-being in Japan and the United States. 


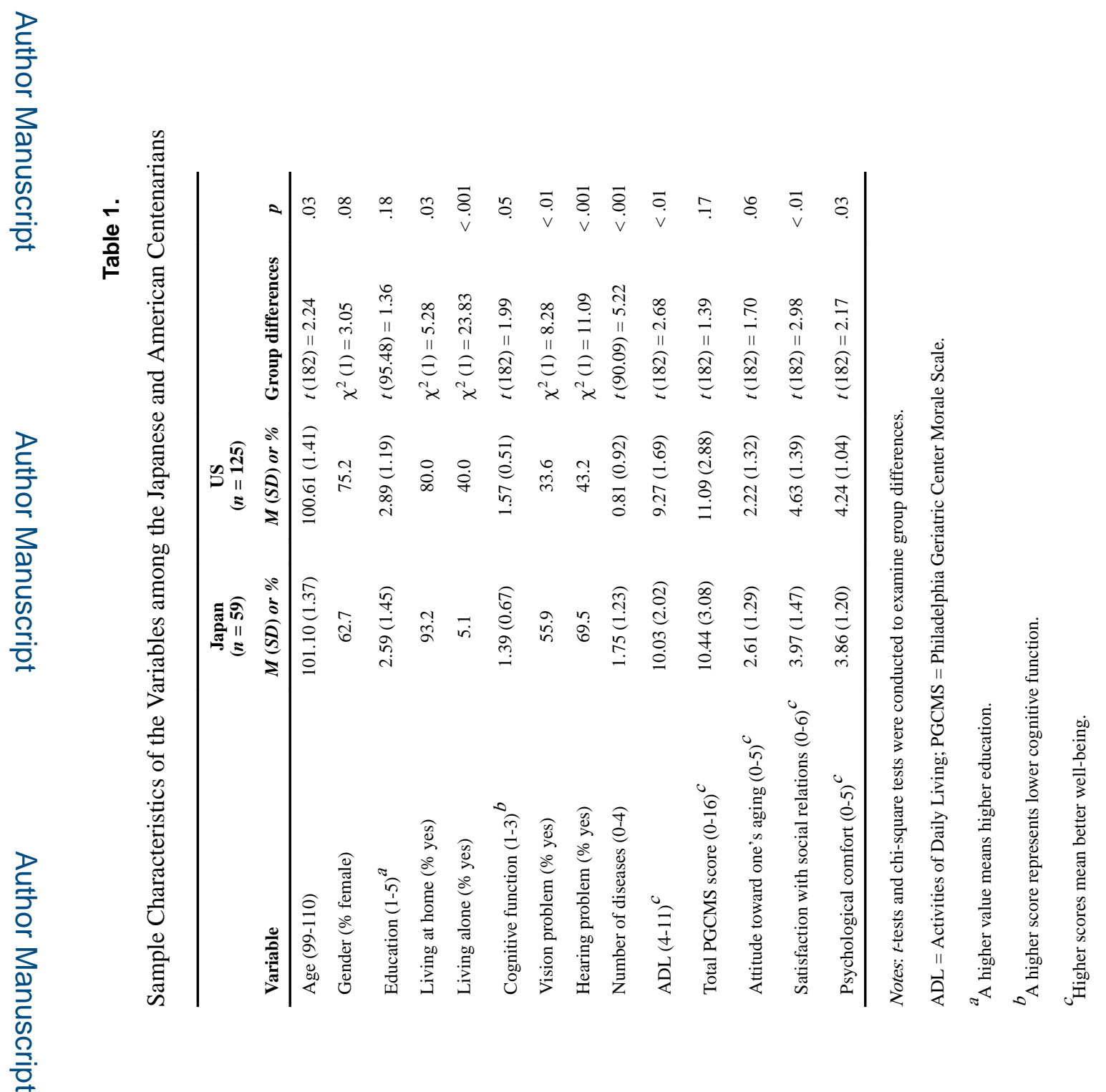


Table 2.

Correlations between the Subjective Well-Being Indicators and Predictors by Country

\begin{tabular}{|c|c|c|c|c|c|c|c|c|}
\hline \multirow[b]{2}{*}{ Variable } & \multicolumn{2}{|c|}{$\begin{array}{c}\text { Total } \\
\text { PGCMS score }\end{array}$} & \multicolumn{2}{|c|}{$\begin{array}{l}\text { Attitude toward } \\
\text { one's aging }\end{array}$} & \multicolumn{2}{|c|}{$\begin{array}{l}\text { Satisfaction } \\
\text { with } \\
\text { social relations }\end{array}$} & \multicolumn{2}{|c|}{$\begin{array}{c}\text { Psychological } \\
\text { comfort }\end{array}$} \\
\hline & $r$ & $p$ & $r$ & $p$ & $r$ & $p$ & $r$ & $p$ \\
\hline & \multicolumn{8}{|c|}{ Japan } \\
\hline Age & -.12 & .38 & -.16 & .23 & -.07 & .62 & -.05 & .73 \\
\hline Gender & -.06 & .65 & .09 & .48 & -.14 & .30 & -.09 & .51 \\
\hline Education & -.09 & .52 & -.05 & .71 & -.02 & .91 & -.15 & .25 \\
\hline Living at home & .08 & .53 & .08 & .57 & .18 & .18 & -.09 & .51 \\
\hline Living alone & -.19 & .16 & -.29 & .03 & -.10 & .45 & -.04 & .77 \\
\hline Cognitive function & .04 & .76 & -.00 & 1.00 & .05 & .72 & .05 & .73 \\
\hline Vision problem & -.11 & .42 & -.03 & .82 & -.11 & .39 & -.10 & .44 \\
\hline Hearing problem & -.23 & .08 & -.17 & .19 & -.12 & .38 & -.26 & .05 \\
\hline Number of diseases & -.07 & .60 & -.03 & .82 & -.09 & .49 & -.04 & .79 \\
\hline \multirow[t]{2}{*}{$\mathrm{ADL}$} & .13 & .31 & -.00 & .99 & .23 & .08 & .07 & .62 \\
\hline & \multicolumn{8}{|c|}{ US } \\
\hline Age & .08 & .38 & .08 & .40 & .09 & .30 & -.00 & .99 \\
\hline Gender & -.05 & .56 & -.03 & .72 & -.05 & .61 & -.05 & .61 \\
\hline Education & -.05 & .61 & -.04 & .69 & -.06 & .55 & -.01 & .91 \\
\hline Living at home & .09 & .31 & .05 & .57 & .11 & .21 & .04 & .67 \\
\hline Living alone & .06 & .55 & .07 & .47 & -.01 & .94 & .08 & .38 \\
\hline Cognitive function & -.21 & .02 & -.09 & .33 & -.18 & .05 & -.24 & $<.01$ \\
\hline Vision problem & -.13 & .14 & -.09 & .31 & -.08 & .37 & -.15 & .10 \\
\hline Hearing problem & -.34 & $<.001$ & -.23 & $<.01$ & -.22 & .01 & -.36 & $<.001$ \\
\hline Number of diseases & -.09 & .32 & -.11 & .22 & .01 & .94 & -.12 & .18 \\
\hline $\mathrm{ADL}$ & .28 & $<.01$ & .20 & .03 & .23 & .01 & .22 & .01 \\
\hline
\end{tabular}

Notes: Correlation coefficients significant at $p<.05$ are reported in bold.

$\mathrm{ADL}=$ Activities of Daily Living; PGCMS = Philadelphia Geriatric Center Morale Scale. 


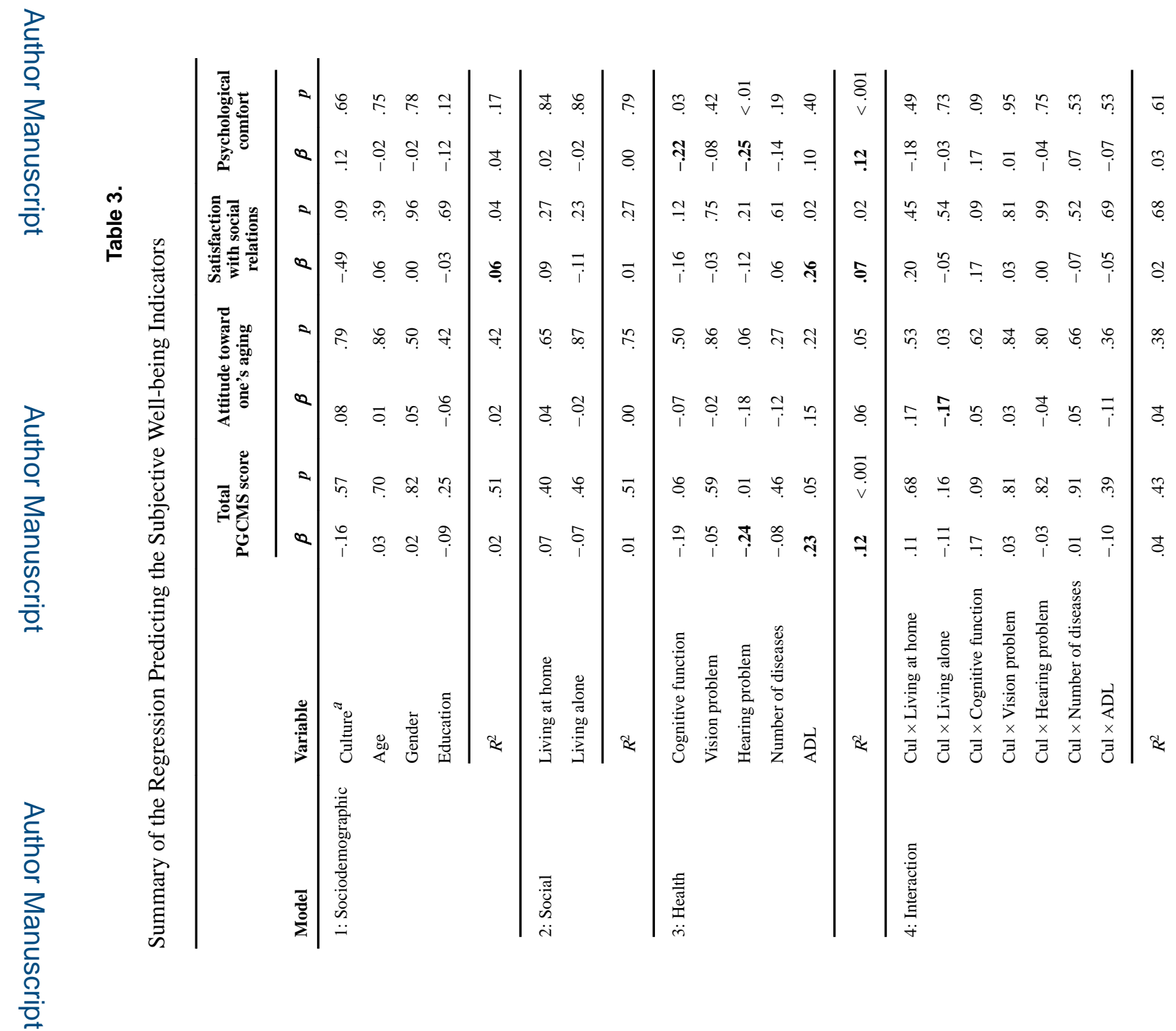




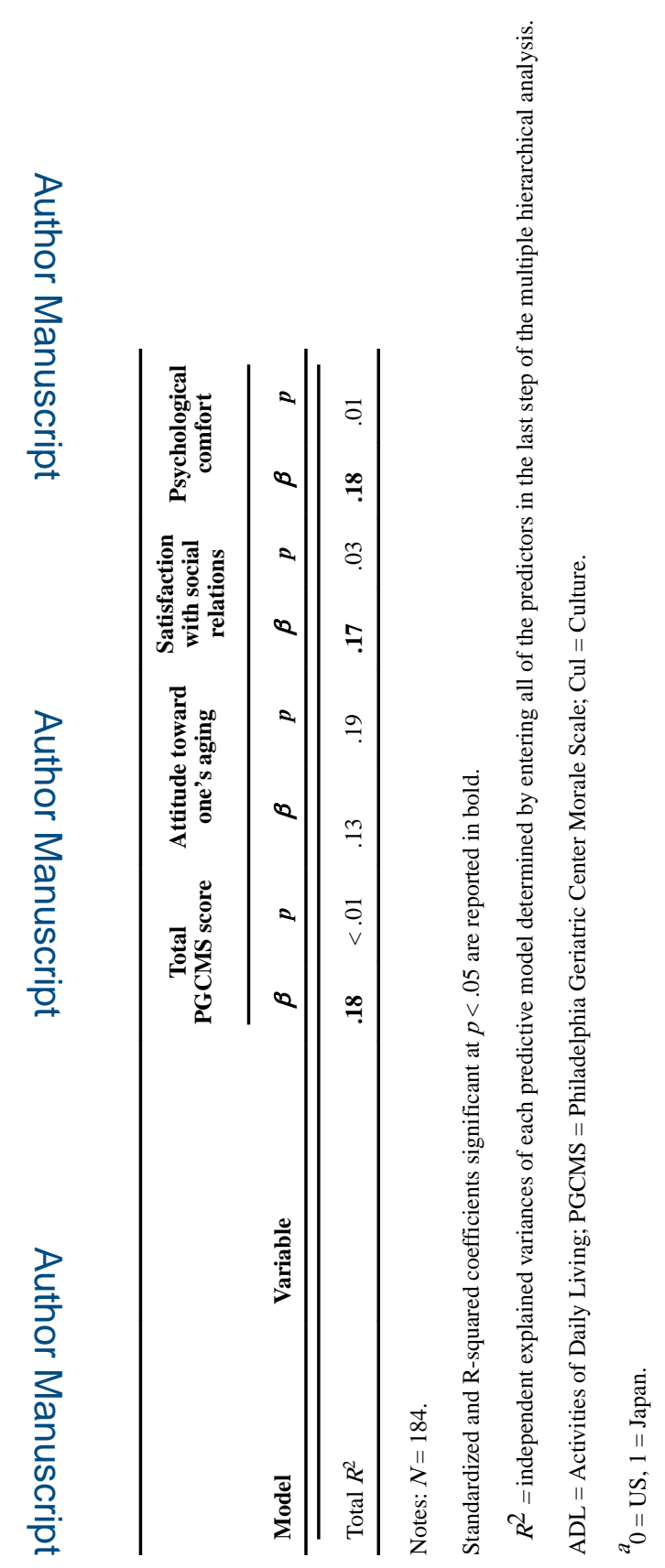

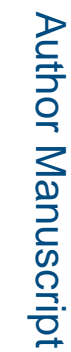

Aging Ment Health. Author manuscript; available in PMC 2019 October 01. 